\title{
Dissociation of covert and overt spatial attention during prehension movements: Selective interference effects
}

\author{
CLAUDIA BONFIGLIOLI and UMBERTO CASTIELLO \\ University of Melbourne, Parkville, Australia
}

\begin{abstract}
In four experiments, the influence of distractor objects on the temporal evolution of the reach-tograsp movement toward a target object (an apple) was examined. In the first experiment, the distractor was another apple, which moved laterally behind the target and occasionally changed direction toward the target, thus becoming the to-be-grasped object. In the second and third experiments, the distractor was a stationary piece of fruit, which sometimes became the to-be-grasped object because of a change in illumination. The fourth experiment was a combination of the first two experiments. In all cases, selective interference effects on the transport and manipulation components were observed only when attention to the distractor was covert rather than overt. It is proposed that covert visuospatial attention selects information about distracting but potentially important stimuli, such that a registration of significance is accomplished without the need to process all available information.
\end{abstract}

Two different modes of attending to relevant stimuli have been described. In the first case, overt changes in the orientation of attention are associated with head and eye movements. In the second case, covert changes in the orientation of attention are usually achieved without eye or body movements (see Umiltà, 1988, for a review).

The link between overt and covert orienting of attention has been a matter of investigation for many years. These processes have been studied mainly with experimental paradigms that present unstructured visual stimuli that require arbitrary manual responses (Posner, 1980 ). There has been very little research in which the influence of each attentional mode on the execution of goaldirected movements has been investigated (Castiello, 1996; Deubel, Schneider, \& Paprotta, 1996, 1998).

Given that attention and eye movement are closely linked in everyday life activities, such as reaching to grasp, it is of interest to investigate how these two processes are organized to produce efficient performance. Can an artificial dissociation produce an imbalance that is reflected in the task that is about to be performed?

Casual observations suggest that close coordination between covert attention and eye movements is necessary for

This work was supported by funds from the Australian Research Council to U.C. C.B. was supported by a grant from the Australian Research Council to U.C. We thank BTS and, in particular, E. D'Amico for technical support. Maria Bulgheroni (Biohelp s.r.1.) is also thanked. Alan Allport, Ada Kritikos, and Paul Maruff are acknowledged for their useful comments on previous versions of this manuscript. Helen Chambers is thanked for her assistance with data acquisition and analysis. C. Bonfiglioli's permanent address is: Dipartimento di Psicologia, Università di Bologna, Italy. Correspondence concerning this article should be addressed to U. Castiello, Department of Psychology, The University of Melbourne, 3052 Parkville, VIC, Australia (e-mail: u.castiello@ psych.unimelb.edu.au). smooth performance, such as during prehensile tasks. It is possible to perform reaching movements toward objects without making a saccade toward them-for example, reaching and grasping an eraser on one's desk while correcting a manuscript, without moving the eyes from the page. Presumably, covert attention is directed toward the eraser, and this is sufficient to allow for the accurate planning of prehension movement (i.e., the position and characteristics of the eraser are coded in such a way as to orient and preshape the hand properly for that action). Nevertheless, sometimes it is necessary to make one or two adjustments, and, if these are not sufficient, it is also necessary to direct the eyes toward the object for final success in the task.

Evidence for the independent action of covert attention during movement comes from tasks and paradigms using distractor objects (Castiello, 1996, Experiments 5 and 6). When subjects were required to perform a concurrent subsidiary task involving the distractor (counting the number of times a laterally placed fruit was illuminated), interference effects were revealed. Specifically, peak grip aperture was influenced by the lateral fruit. If, for example, the central target fruit was a cherry, the amplitude of peak grip aperture was greater when the distractor fruit was an apple than when it was a banana. Conversely, the amplitude of peak grip aperture for the grasp of an apple was less when the lateral fruit was a cherry than when it was a mandarin. The distractor thus appeared to disturb the correct output. Interference was found only when covert attention presumably was directed to a lateral nontarget fruit and was dissociated from eye movements. In other words, the programming of an arm movement can be influenced by attended information in the visual field, and this is independent of eye movements.

The first prediction for the present study is that the covert orienting of attention toward a nontarget distrac- 
tor will modify the pattern of prehension to a relevant object, as compared with a condition in which eye movements are unrestricted.

According to the channel hypothesis proposed by Jeannerod $(1981,1984)$, prehension movements are subserved by two functionally independent channels - the transport component and the manipulation component. The transport channel extracts information regarding the spatial location of the object and allows for the transformation of this information into commands that are appropriate for bringing the hand toward the object. The manipulation channel extracts information regarding the size and shape of the object, thus allowing the implementation of the distal movement pattern necessary to grasp the object. Jeannerod's $(1981,1994)$ idea was quite original, because it embodied the notion that the same stimulus is processed independently and in parallel for the control of different motor acts. A notion, however, that is still a matter of debate is the impermeability of one component to the information related to the other component. Recent studies on prehension to an object presented in isolation have challenged such a concept. In these studies, it was found, for example, that object size not only influences parameterization of the manipulation component but also that of the transport component (see, e.g., Bootsma \& van Wieringen, 1992; Castiello, Paulignan, \& Jeannerod, 1991; Gentilucci et al., 1991; Gentilucci, Chieffi, Scarpa, \& Castiello, 1992), whereas object distance influences not only the transport component but also the manipulation component (Jakobson \& Goodale, 1991).

In the present study, the notion of impermeability is tackled from a different perspective. That is, impermeability is not verified by looking at the effects that different characteristics of the same object have on the channel not devoted to their programming, but it is asked whether a second object, considered as a distractor; can selectively influence the transport or the manipulation component. Further, in this study, the question of whether the distractor could independently influence either the transport or the manipulation component of a prehension movement to a target object when covert attention is directed on it is investigated.

\section{EXPERIMENT 1}

In this experiment, the goal of the movement and the distractor were of the same type (i.e., an apple). The distractor object could move from left to right, and, if it crossed a red mark positioned along the track, the subject had to grasp it. If the distractor did not cross the red mark (i.e., stopped before it), the target (the same apple) needed to be grasped. Thus, the main feature of the experimental manipulation was that the distractor could become, at any instant, the target for the reach. In other words, the direction was either to grasp the static apple when the distractor stopped before the red mark or to grasp the moving apple if it passed the red mark. Two conditions were tested. In one condition, the subjects were required to keep their eyes fixated on the target. In the other condition, eye movements were permitted. The aim of the present experiment was to highlight changes only at the level of the transport component. It was predicted that there would be no changes to the manipulation component, given that the distractor was identical to the target (i.e., it required the same type of parameterization as the target).

\section{Method}

\section{Subjects}

Eight students (4 women and 4 men, 21-32 years of age) volunteered to participate in this experiment. All were right-handed according to the Edinburgh Inventory (Oldfield, 1971), reported normal or corrected-to-normal vision, and were ignorant as to the purpose of the experiment. Each subject attended two experimental sessions of approximately l-h duration. These sessions took place on 2 separate days.

\section{Apparatus and Materials}

The experiment was conducted under normal room-lit conditions. Details of the experimental setup are shown in Figure 1. The subject was seated in front of the table working surface $(1 \times 1 \mathrm{~m})$. Prior to each trial, the subject placed the right hand on the table in the mid-sagittal plane, $15 \mathrm{~cm}$ from the thorax. In this position, the shoulder was flexed $\left(5^{\circ}-10^{\circ}\right)$, the elbow flexed, the forearm semipronated, and the wrist was in $10^{\circ}-15^{\circ}$ of extension. The index finger and thumb were held gently opposed, and the ulnar border of the hand rested on a pressure-sensitive starting switch.

\section{Design}

Two conditions were considered.

Condition $\mathbf{A}$. In this condition, the target object and the distractor object were of the same type (i.e., an apple). A piece of fruit was placed on a carrier ( $9 \mathrm{~cm}$ in length, $5 \mathrm{~cm}$ in depth, and $2 \mathrm{~cm}$ in height) that could travel along a T-shaped track, embedded in a table surface. A concave depression in the top surface of the carrier served to keep the fruit stable during the displacement. Such a device was positioned $0.50 \mathrm{~m}$ from the target stimulus (see Figure 1). The track allowed the carrier, driven by an electric motor, to displace the fruitdistractor from left to right at variable speed $(\sim 40-60 \mathrm{~cm} / \mathrm{sec})$. The subjects could see the fruit moving but not the carrier. Sometimes the carrier arrived at the $\mathrm{T}$ junction and then briskly deviated toward the target. Ten centimeters beyond this deviation, a red mark $(5 \times 0.5 \mathrm{~cm})$ was positioned along this part of the track. If the carrier crossed the red mark, the subject had to grasp the distractor fruit. No instructions as to how to perform the movement in terms of speed and accuracy were given. Thus, the main feature of the experimental manipulation was that the distractor became relevant for the subject in a particular manner--namely, that at any instant it might become the target for the (perturbed) reach. The time course of deviation of the carrier varied with the speed: $250 \mathrm{msec}$ at $40 \mathrm{~cm} / \mathrm{sec}$ and $166 \mathrm{msec}$ at $60 \mathrm{~cm} / \mathrm{sec}$. However, there were also intermediate values for speeds between 40 and $60 \mathrm{~cm} / \mathrm{sec}$. The average time course was $205 \mathrm{msec}$. The subjects were required to keep their eyes fixated on the target. Eye movements were monitored (see the Recording section).

Condition B. This condition was similar in all respects to Condition A, except that the subjects were told that they could move their eyes without any constraint.

\section{Recording}

Reflective passive markers $(0.25 \mathrm{~cm}$ in diameter $)$ were attached to the reaching limb (transport component) at the wrist - radial aspect of the distal styloid process of the radius-and to the hand (ma- 


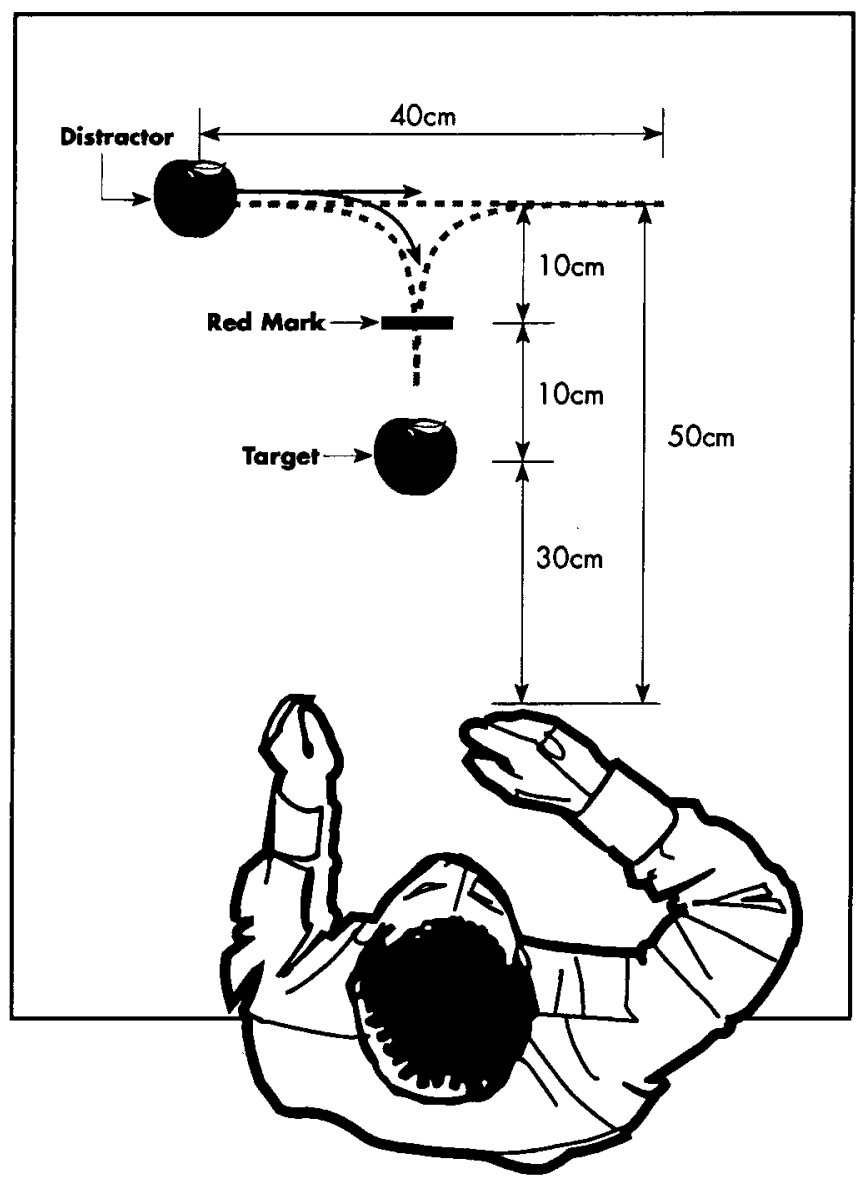

Figure 1. Experimental setup utilized in Experiment 1.

nipulation component) at the index finger - radial side of the nailand the thumb-ulnar side of the nail. Movements were recorded with the Elite system (Ferrigno \& Pedotti, 1985). This consisted of two infrared cameras (sampling rate, $100 \mathrm{~Hz}$ ) inclined at an angle of $30^{\circ}$ to the vertical and placed $3 \mathrm{~m}$ in front of the table and $3 \mathrm{~m}$ apart. The calibrated working space was a parallelepiped (length of $60 \mathrm{~cm}$, breadth of $30 \mathrm{~cm}$, height of $60 \mathrm{~cm}$ ), from which the spatial error measured from stationary and moving stimuli was $0.04 \mathrm{~mm}$. Calibration was performed using a grid of 25 markers $(5 \times 5)$. The centroid of each marker was placed $15 \mathrm{~cm}$ from that of another. Using the procedure of Haggard and Wing (1990), the mean length of a bar with two markers attached $15 \mathrm{~cm}$ apart, as reconstructed from the Elite data, was found to be $14.7 \mathrm{~cm}(S D, 0.22 \mathrm{~cm})$. Coordinates of the markers were reconstructed with an accuracy of $1 / 3,000$ over the field of view and sent to a host computer (PC 486). The $S D$ of the reconstruction error was $1 / 3,000$ for the vertical $(y)$ axis and 1.4/3,000 for the two horizontal ( $x$ and $z$ ) axes.

Horizontal and vertical eye positions were monitored with an infrared corneal reflection system (sampling frequency, $120 \mathrm{~Hz}$ ) mounted in eyeglass frames. Trials in which eye movement was in excess of $1^{\circ}$ of visual angle (vertical and horizontal) were detected, discarded, and replaced.

\section{Procedure}

This experiment consisted of two sessions, in which two different experimental conditions were tested. The order of sessions was counterbalanced across subjects. Experimentation continued over a 2-day period.

Condition $\mathbf{A}$. The carrier traveled at variable speeds and started from different positions, with the fruit-distractor on the top. In order to avoid the change in speed or direction being detected from the sound of the carriage, the subject had earphones. At a randomized time $(2,000-6,000 \mathrm{msec})$, a starting tone (through the earphones) was given after the carrier started to travel. The subject was required to reach for and grasp the target fruit and to bring it back to the position in which the hand had rested before movement initiation. However, for $20 \%$ of the total number of trials in which the carrier was moving (100), the carrier, upon arriving at the $T$ junction, briskly deviated toward the subject and toward the target stimulus. The subject was required to grasp the oncoming fruit distractor if the carrier crossed the red mark positioned $10 \mathrm{~cm}$ from the deviation point, while keeping the eyes fixed on the target fruit (see Figure 1). In other words, the subject had to monitor the carrier covertly throughout the trial, to detect a possible change of direction toward the red mark. Thirty trials without any movement of the distractor were also presented. With respect to the target object, the distractor was positioned 10 times on the right, 10 times central, and 10 times on the left. In summary, there were 80 trials (control) in which the carrier was moving but did not deviate (i.e., it traveled from left to right and from right to left between the two ends of the T), 20 trials (perturbed) in which the carrier deviated toward the target fruit, and 30 trials (blocked) without movement. To this total of 
130 trials, 10 trials were added in which no distractor was presented (Table 1). The sequence of carrier directions was preprogrammed and counterbalanced. Trials in which eye movements occurred were discarded and successively repeated. Experimentation continued until the required number of trials had been performed.

Condition B. The procedure and the task for Condition B was exactly the same as for Condition $\mathrm{A}$, but eye movements were not constrained. In other words, the subjects were required to perform exactly the same task as for Condition A, except that they could move their eyes without any limitation.

\section{Data Processing}

The ELIGRASP (B $|\mathrm{T}| \mathrm{S} \mid$, 1994) software package was used to assess the data. This gave a three-dimensional reconstruction of the marker positions. The data were then filtered, using a FIR linear filter - transition band of $1 \mathrm{~Hz}$ (sharpening variable $=2$; D'Amico \& Ferrigno, 1990, 1992) and a cutoff frequency of $10 \mathrm{~Hz}$. The transport component was assessed by analyzing velocity and acceleration profiles of the wrist marker. The manipulation component was assessed by analyzing each of the hand markers and the distance between these two markers. The velocity of the opening and closing of the digits was also assessed. Movement initiation time - so called because no emphasis was placed on a rapid response-was taken from the release of the starting switch after a tone sounded. The end of the movement was taken to be the time at which the fingers closed on the fruit and there was no further change in the distance between the index finger and the thumb. The period following this was not assessed. The dependent variables were initiation time, movement duration, and the kinematic variables: for the transport component, the time to peak velocity, the time to peak acceleration, the time to peak deceleration of the wrist marker, and the amplitudes of these peaks; and, for the manipulations component, the time to peak grip aperture, the time to peak grip velocity, and the amplitudes of the aperture and velocity peaks. Each temporal value was also calculated as a percentage of movement duration (relative values).

\section{Results and Discussion}

Initiation time, movement duration, and kinematic variables (absolute and relative) were analyzed with a $2 \times 2$ repeated measures analysis of variance (ANOVA) with condition (A and B) as the first factor and type of distractor (no distractor, moving, and stationary) as the second factor.

On nine occasions for Condition A and across all the subjects, the distractor object had to be caught because the red mark was crossed over. Given the limited number of values, these data have not been involved in any of the following analyses, but they were analyzed (see the Appendix). In addition, the trials in which the distractor object deviated toward the target object but the subject grasped the target before the distractor crossed the red mark were compared with the control trials. No differ- ences for any of the dependent measures were found. In order to compare the same number of trials for the nodistractor $(n=10)$, the stationary distractor (blocked trials; $n=30$ ), and the moving distractor (control and perturbed trials; $n=100$ ) sets, only 10 trials for the moving distractor situation were chosen, randomly by means of a computer software. The same was done for the stationary distractor condition, given that, for these trials, no differences with respect to distractor position were found.

The condition $\times$ type of distractor interaction was significant for a number of dependent measures. For Condition A (eye movements monitored, eyes fixated on the target), differences between the moving and the stationary distractor conditions were found (see Table 2). However, for Condition B (eye movements not restricted), no differences were found, except for increased variability for some dependent measures for the moving distractor, with respect to the stationary and the no-distractor sets (see Table 2 and Figure 2). Also, no differences were found between the no-distractor and the stationary distractor sets for Condition $A$ and between the three distractor conditions in Condition B (see Table 2).

For the sake of brevity, only the results for Condition A and the mean values for the moving and stationary distractor will be reported in the text. For the other values and statistics, please refer to Table 2 .

Initiation time, movement time, and the kinematic results indicated that movement was affected by the presence of the distractor. Initiation time was longer when the distractor was moving, as compared with when it was stationary ( 381 vs. $367 \mathrm{msec}$ ). Also, movement time was influenced by the moving distractor. The time taken to reach and grasp the stationary target object in the presence of a moving distractor object was longer than the time taken to reach and grasp the stationary target object in the presence of a stationary distractor object ( $797 \mathrm{vs}$. $748 \mathrm{msec}$ ). The time of peak velocity, acceleration, and deceleration occurred later for the moving than for the stationary distractor condition, in absolute and relative terms (time to peak velocity-absolute, 366 vs. $312 \mathrm{msec}$; relative, $46 \%$ vs. $41 \%$; time to peak acceleration-absolute, 271 vs. $231 \mathrm{msec}$; relative, $34 \%$ vs. $31 \%$; time to peak deceleration-absolute, 533 vs. $466 \mathrm{msec}$; relative, $67 \%$ vs. $62 \%$ ). Also, the amplitudes of the velocity, acceleration, and deceleration peaks were all lower for the movements performed in the presence of the moving distractor than for those performed in the presence of the stationary distractor (amplitude peak velocity, $705 \mathrm{vs}$. $841 \mathrm{~mm} / \mathrm{sec}$; amplitude peak acceleration, 4,083 vs.

Table 1

Number of Trials for the Moving Distractor, Stationary Distractor, and No-Distractor Sets in Experiment 1

Moving Distractor

\begin{tabular}{|c|c|c|c|c|c|c|}
\hline & \multicolumn{2}{|c|}{ Moving Distractor } & \multirow{2}{*}{\multicolumn{3}{|c|}{ Stationary Distractor }} & \multirow[b]{3}{*}{ No Distractor } \\
\hline & \multirow{2}{*}{$\begin{array}{c}\text { Deviation } \\
\text { (Perturbed Trials) }\end{array}$} & \multirow{2}{*}{$\begin{array}{c}\text { No Deviation } \\
\text { (Control Trials) }\end{array}$} & & & & \\
\hline & & & Left & Center & Right & \\
\hline No. of trials & 20 & 80 & 10 & 10 & 10 & 10 \\
\hline
\end{tabular}


Table 2

Initiation Time, Movement Duration, and Kinematic Parameters for Condition A and Condition B in Moving Distractor, No-Distractor, and Stationary Distractor Conditions in Experiment 1

\begin{tabular}{|c|c|c|c|c|c|c|c|c|c|c|c|c|c|}
\hline \multirow[b]{3}{*}{ Dependent Variables } & \multicolumn{7}{|c|}{ Condition A } & \multicolumn{6}{|c|}{ Condition B } \\
\hline & \multicolumn{2}{|c|}{$\begin{array}{l}\text { Moving } \\
\text { Distractor }\end{array}$} & \multicolumn{2}{|c|}{$\begin{array}{c}\text { No } \\
\text { Distractor }\end{array}$} & \multicolumn{2}{|c|}{$\begin{array}{l}\text { Stationary } \\
\text { Distractor }\end{array}$} & \multirow[b]{2}{*}{ Statistical Values } & \multicolumn{2}{|c|}{$\begin{array}{l}\text { Moving } \\
\text { Distractor }\end{array}$} & \multicolumn{2}{|c|}{$\begin{array}{c}\text { No } \\
\text { Distractor } \\
\end{array}$} & \multicolumn{2}{|c|}{$\begin{array}{l}\text { Stationary } \\
\text { Distractor }\end{array}$} \\
\hline & $M$ & $S D$ & $M$ & $S D$ & $M$ & $S D$ & & $M$ & $S D$ & $M$ & $S D$ & $M$ & $S D$ \\
\hline Initiation time (msec) & 381 & 60 & 369 & 40 & 367 & 40 & $F(1,7)=19.05, p<.0001$ & 361 & 64 & 361 & 36 & 359 & 41 \\
\hline Movement duration (msec) & 797 & 121 & 756 & 84 & 748 & 86 & $F(1,7)=8.65, p<.05$ & 739 & 113 & 744 & 72 & 729 & 84 \\
\hline \multicolumn{14}{|l|}{ Transport component } \\
\hline Time to peak velocity $(\mathrm{mm} / \mathrm{sec})$ & 366 & 35 & 315 & 32 & 312 & 35 & $F(1,7)=17.06, p<.0001$ & 313 & 34 & 320 & 21 & 318 & 29 \\
\hline Time to peak velocity $(\%)$ & 46 & 6 & 41 & 3 & 41 & 5 & $F(1,7)=25.01, p<.0001$ & 42 & 4 & 43 & 3 & 43 & 5 \\
\hline Time to peak acceleration (msec) & 271 & 26 & 234 & 22 & 231 & 27 & $F(1,7)=10.89, p<.001$ & 231 & 28 & 239 & 28 & 232 & 32 \\
\hline Time to peak acceleration $(\%)$ & 34 & 4 & 30 & 2 & 31 & 3 & $F(1,7)=9.32, p<.001$ & 31 & 2 & 32 & 4 & 31 & 3 \\
\hline Time to peak deceleration (msec) & 533 & 55 & 477 & 51 & 466 & 54 & $F(1,7)=19.02, p<.001$ & 471 & 58 & 478 & 52 & 464 & 55 \\
\hline Time to peak deceleration $(\%)$ & 67 & 4 & 63 & 4 & 62 & 5 & $F(1,7)=6.01, p<.05$ & 63 & 5 & 64 & 4 & 63 & 6 \\
\hline Amplitude peak velocity $(\mathrm{mm} / \mathrm{sec})$ & 705 & 145 & 884 & 100 & 841 & 137 & $F(1,7)=36.54, p<.0001$ & 877 & 132 & 900 & 88 & 881 & 165 \\
\hline Amplitude peak acceleration $\left(\mathrm{mm} / \mathrm{sec}^{2}\right)$ & 4,083 & 543 & 6,002 & 598 & 5,417 & 624 & $F(1,7)=40.04, p<.0001$ & 5,878 & 722 & 5,843 & 622 & 5,727 & 612 \\
\hline Amplitude peak deceleration $\left(\mathrm{mm} / \mathrm{sec}^{2}\right)$ & 3,824 & 444 & 5,678 & 657 & 5,286 & 433 & $F(1,7)=29.06, p<.0001$ & 5,422 & 654 & 5,501 & 555 & 5,428 & 613 \\
\hline \multicolumn{14}{|c|}{ Manipulation component } \\
\hline Time to maximum grip aperture (msec) & 517 & 47 & 488 & 55 & 485 & 68 & n.s. & 461 & 63 & 473 & 49 & 458 & 66 \\
\hline Time to maximum grip aperture $(\%)$ & 64 & 5 & 64 & 4 & 64 & 7 & n.s. & 62 & 4 & 63 & 5 & 62 & 6 \\
\hline Velocity of finger aperture $(\mathrm{mm} / \mathrm{sec})$ & 368 & 40 & 354 & 36 & 371 & 38 & n.s. & 402 & 39 & 387 & 39 & 391 & 41 \\
\hline Amplitude grip aperture $(\mathrm{mm})$ & 107 & 2 & 108 & 3 & 110 & 2 & n.s. & 110 & 4 & 111 & 1 & 110 & 4 \\
\hline
\end{tabular}

Note-Statistical values for Condition B were not significant.

$5,417 \mathrm{~mm} / \mathrm{sec}^{2}$; amplitude peak deceleration, $3,824 \mathrm{vs}$. $5,286 \mathrm{~mm} / \mathrm{sec}^{2}$ ).

The results for the manipulation component were in accordance with those from previous studies of reaching to grasp (Castiello, 1996; Castiello, Bennett, \& Stelmach, 1993; Jeannerod, 1981, 1984; Marteniuk, Leavitt, MacKenzie, \& Athenes, 1990). As an example, for the moving condition, maximum peak of grip aperture occurred at $64 \%$ of movement duration, and the mean amplitude was $107 \mathrm{~mm}$. No differences were found with respect to the other distractor conditions (see Table 2).

Of particular note, in the latter condition, was that the distractor was moving. That is, there was a need to attend in some way to the distractor for the control of the action. This was because, sometimes, the distractor could become the target. As a consequence, this situation revealed effects that were not seen when the distractor was passive (see, e.g., Castiello, 1996). We suggest that, in this experiment, the target of the reach-to-grasp movement and the active distractor were competing for control (Duncan \& Desimone, 1995).

Interestingly, it was only the transport component that was predominantly affected by the presence of the moving distractor. Previous research has demonstrated that the manipulation component could be influenced by changes relative to the transport component (e.g., distance or position; Bootsma \& van Wieringen, 1992; Castiello et al., 1991; Gentilucci et al., 1992; Haggard, 1994; Jakobson \& Goodale, 1991; Marteniuk et al., 1990; Paulignan, MacKenzie, Marteniuk, \& Jeannerod, 1991; Wallace \& Weeks, 1988; Wing, Turton, \& Fraser, 1986). However, for the present experiment, it could be suggested that there was no need for a change at the manipulation component level, given that the type of prehension for the distractor object was the same as that for the target object.

In summary when the eyes were fixed on the target, covert mechanisms of spatial attention were needed to monitor the location of the distractor. The increase in the level of disturbance on some of the dependent measures for this condition could be due to the dissociation between eye fixation and attention, thus to a reduction in the efficiency of control. Conversely, when eye movements were not constrained, the overt mechanisms of attention may have reduced the level of interference. The result is an optimization for the motor output. In this respect, it is important to note that, when eye movements were allowed, eye movements were actually made. As can be seen in the upper panel of Figure 3, eye $x-y$ trajectories from one representative subject trial show that, during the task, the eyes were moving from the target to the distractor position. Given that interference effects related to this overt/covert attention dissociation are also common for the next three experiments, further debate on this point, and possible interpretative models, are attempted in the General Discussion section.

\section{EXPERIMENT 2}

Experiment 1 showed that, when subjects attended covertly to the distractor, only the transport component was influenced, whereas no changes for the manipulation component were found. One reason for this could be that the moving distractor was relevant mainly to the transport component of the reach-to-grasp movement, because the type of grasp required by the distractor object was the same as the type of grasp required by the tar- 
Movement Duration

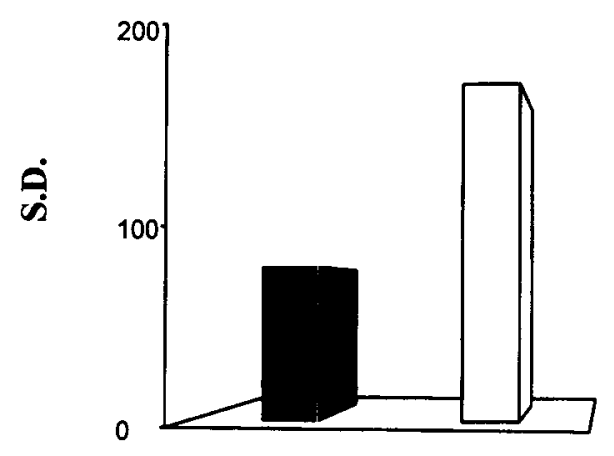

Grip Velocity

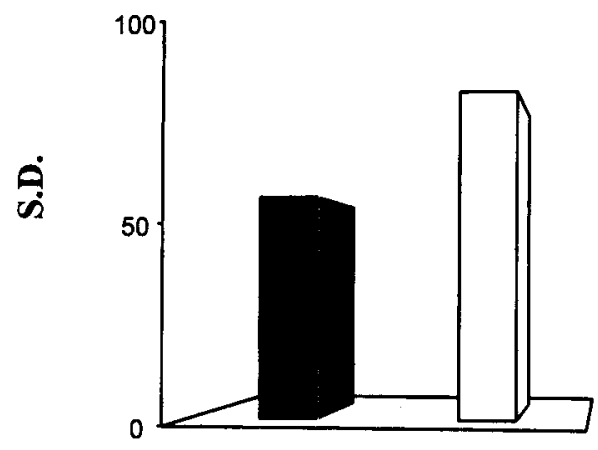

Session A
Initiation Time

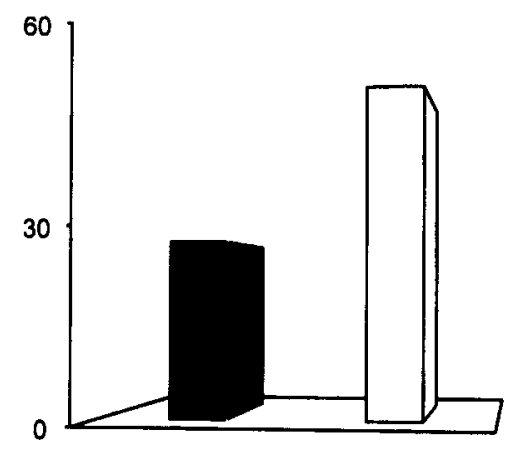

Time Max. Grip Aperture

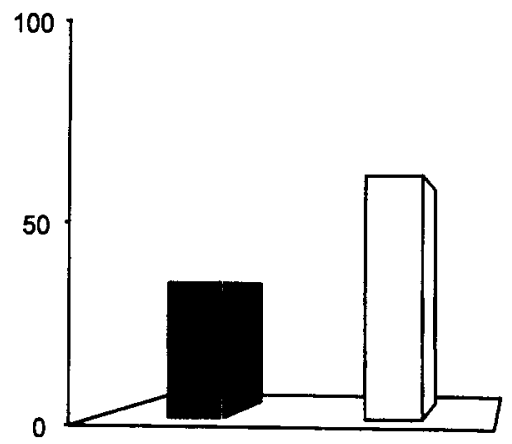

Figure 2. Differences in intertrial variability between Condition $A$ and Condition $B$ in Experiment 1 ( $S D$, standard deviation).

get object. Thus, no changes for the manipulation component were required. Instead, the continuous movement of the distractor determined competition for processing resources in the transport component channel, given that it had to quickly adapt to a brisk change in direction, if it occurred. By following this line of reasoning in Experiment 2, the question of whether it was possible to alter the manipulation component of the reach-to-grasp movement by changing different aspects of the distractor stimulus was investigated. Thus, it could be predicted that different object characteristics are considered selectively in relation to the action-control modification required by the distractor. In this experiment, the target and the distractor objects were different (target, an apple; distractor, a raspberry; this combination remained fixed), each requiring a different type of grasp. The distractor was a stationary piece of fruit (a raspberry), which sometimes became the to-be-grasped object because of a sudden change in illumination. In order to minimize changes for the transport component, the distractor and the target objects were adjacent to one another (see Figure 3). Thus, it was mainly the manipulation component that might need to be changed.

\section{Method}

\section{Subjects}

Eight students ( 4 women and 4 men, 22-25 years of age) volunteered to participate in this experiment; none had participated in the previous experiment. They showed the same general characteristics as did the subjects of the previous experiment. Each subject attended two experimental sessions of $1-h$ duration, in which two conditions ( $\mathrm{A}$ and $\mathrm{B}$ ) were administered.

\section{Apparatus and Materials}

The apparatus and materials were the same as those described for Experiment 1, except for the changes highlighted in the Design section.

\section{Design}

Two conditions were considered.

Condition $\mathbf{A}$. In this experiment, the distractor (a raspberry) was placed on top of a wood block $(7 \mathrm{~cm}$ in height $\times 10 \mathrm{~cm}$ in depth $\times$ $2 \mathrm{~cm}$ in width). A red mark was positioned on the wood block. $1.5 \mathrm{~cm}$ from the edge. A spotlight was located $10 \mathrm{~cm}$ laterally to the wood 


\section{Experiment 1}

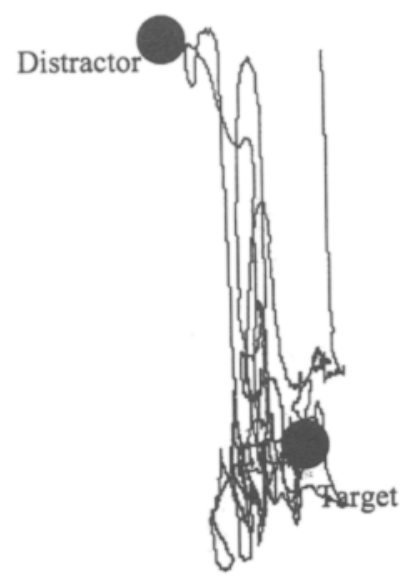

\section{Experiment 2}

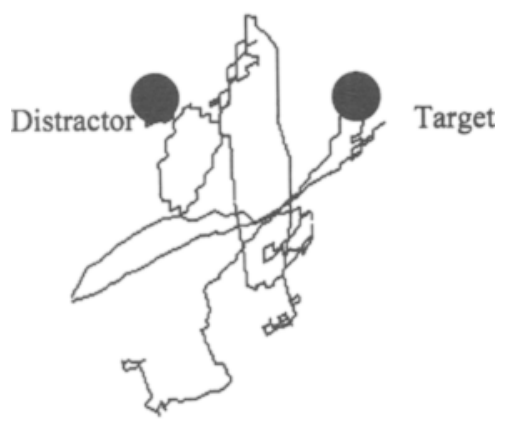

Figure 3. Eye $x-y$ trajectory for a representative trial (Subject: J.T.) for the moving distractor in Condition $B$ for Experiments 1 and 2 . This figure shows that the eyes were constantly moving from one object to the other (and to other positions) during the prehension task.

block and was calibrated to emit a very thin beam of light (see Figure 4). This beam could move at different intervals of time (from 100 to $800 \mathrm{msec}$ ) from the bottom, upwards toward the edge of the wood block, and vice versa. The color of the beam was yellow, and each beam projected a stripe of light on the wood block. The position of the spotlight was regulated by a sensor device positioned underneath the hinge of the spotlight. Eye movements were monitored as they were for Condition $\mathrm{A}$ in Experiment 1 .

Condition B. This condition was similar in all respects to Condition A, except that eye movements were not constrained.

\section{Procedure}

The experiment consisted of two sessions in which two different experimental conditions were tested. The order of sessions was counterbalanced across subjects. Experimentation took place over a 2-day period.

Condition A. The experiment started when the spotlight illuminated the wood block from the bottom upwards toward the edge or from the edge toward the bottom. A starting tone was given, and the subject was required to reach for and grasp the target fruit and to bring it back to the starting position. The starting tone was given at a randomized time after the beam started to illuminate the wooden block $(2,000-6,000 \mathrm{msec})$. However, for $20 \%$ of the 100 trials in which the spotlight was active, the ray of light changed its normal distribution and suddenly illuminated the wood block over the red mark. In this case, the subject was required to grasp the raspberry positioned on the top of the wood block. There were 10 trials in which the wooden block and the raspberry were presented but the spotlight was never turned on (stationary distractor), 80 control trials in which the spotlight gave successive beams running up and down the block above or below the red mark (control trials), 20 trials in which the spotlight crossed the red mark on the wooden block (moving distractor), and 10 trials in which neither the wood block nor the raspberry was presented (no distractor). For this latter condition, the wood block and the spotlight were momentarily removed. In summary, the procedure was as follows: Subjects normally had to grasp the apple, but, if the light from above or below passed the red mark, the subjects had to grasp the raspberry.

For the sake of clarity, the name of the different distractor conditions have been kept similar to those adopted in the previous experiment, even if, in the present experiment, there is not actually a moving distractor condition.

Condition B. The procedure and the task for Condition B were exactly the same as those for Condition $A$, but eye movements were not constrained. In other words, the subjects were required to perform exactly the same task as that for Condition A, except that they could move their eyes without any limitation.

\section{Results and Discussion}

Initiation time, movement duration, and kinematic variables were analyzed with an ANOVA, with condition (A and B) and type of distractor (no distractor, moving, stationary) as factors. For this experiment, when the eye movements were not constrained, no significant effects for any of the dependent measures were found. Further, no differences between the no-distractor and the stationary conditions were found (see Table 3 ). Thus, for the sake of brevity, only results relating to the difference between the moving and the stationary distractors for Condition $\mathrm{A}$ will be reported in the text. For the other values and statistics, refer to Table 2.

In all occasions, the subjects reached for the target before the light passed the red mark. Initiation time and movement duration were longer for the moving condition than for the stationary condition (initiation time, $397 \mathrm{vs.}$ $354 \mathrm{msec}$; movement duration, 812 vs. $732 \mathrm{msec}$; see Figure 5).

In absolute terms, the kinematic parameters of the transport component were delayed by the presence of the activated spotlight (see Table 3 ). However, in relative terms, no differences between the moving and the other distractor conditions were found. This means that the moving distractor affected the absolute timing of the action but not its intrinsic kinematic parameterization. For example, time to peak velocity was reached significantly later in the moving than in the stationary distractor condition ( 341 vs. $300 \mathrm{msec}$ ), but in relative terms (as a percentage of movement duration), it occurred at the same time $(42 \%$ vs. $41 \%)$. The same applies for times to peak acceleration and deceleration (see Table 3 and Figure 5).

A noticeable finding was that the amplitude of the maximum grip aperture and of the velocity of finger aperture, measured for the moving distractor, were smaller 

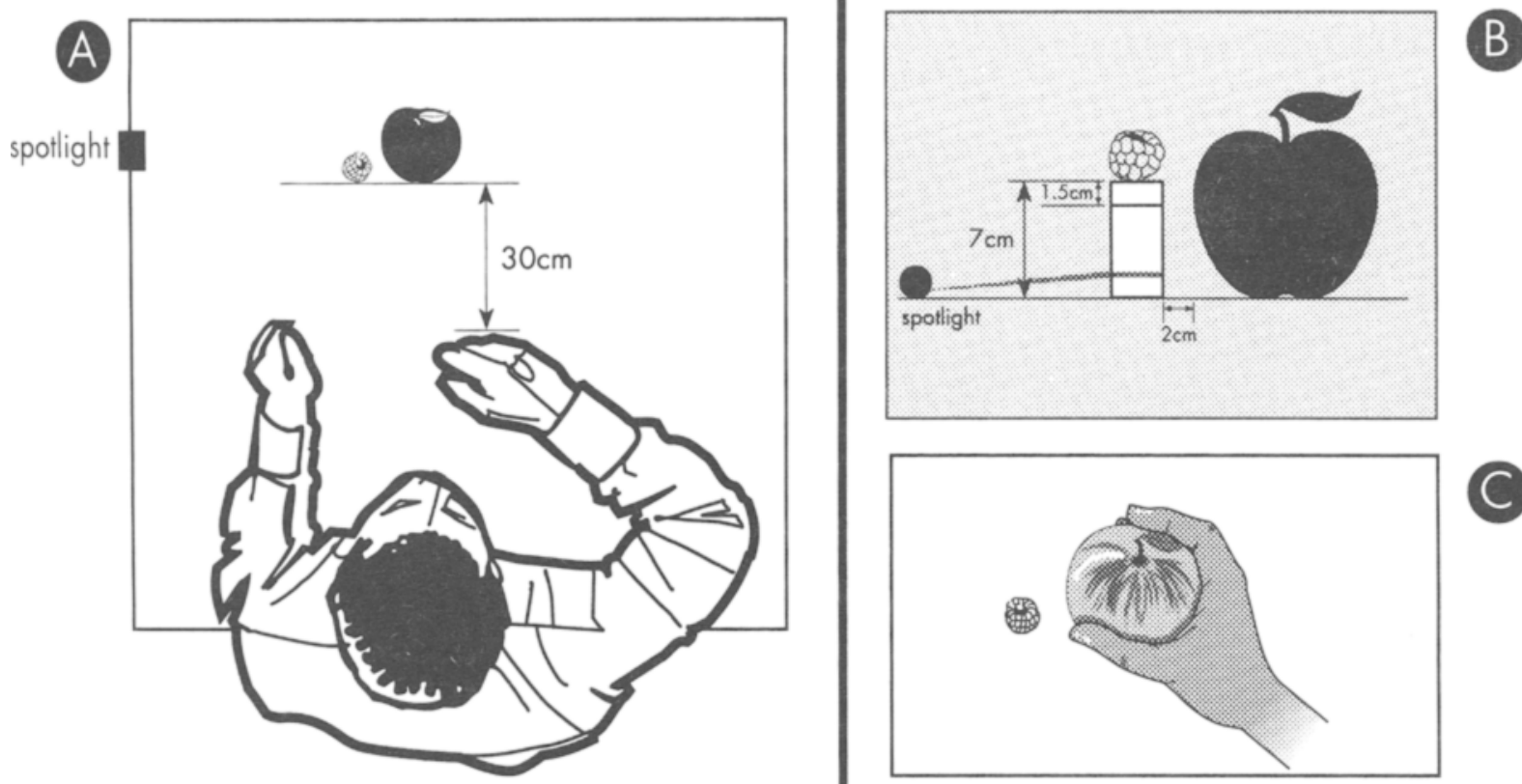

Figure 4. Panel A: experimental setup utilized in Experiment 2. Panel B: side view of the experimental setup. Panel C shows that the distractor did not interfere physically with the movement directed toward the target.

than that measured for the stationary distractor sets (amplitude grip aperture, $86 \mathrm{vs} .109 \mathrm{~mm}$; velocity of finger aperture, 314 vs. $456 \mathrm{~mm} / \mathrm{sec}$ ). Thus, the possibility that a different size of grasp could be required influenced the aperture for the target object. In addition, the time of maximum grip aperture occurs later, in both absolute and relative terms, for the moving than for the stationary distractor condition (absolute, 544 vs. $469 \mathrm{msec}$; relative, $67 \%$ vs. $64 \%$; see Figure 5 ).
Once again, for the moving distractor, it is suggested that the distractor needed to be monitored for a possible modification of the action. This situation revealed effects that were not seen with only a concurrent passive distractor or in the no-distractor condition. However, it is of some interest that it was only the manipulation component that was affected by the presence of the distractor. Possibly, there was no need for the transport component to be reorganized significantly, given that the

Table 3

Initiation Time, Movement Duration, and Kinematic Parameters in the Moving Distractor, No-Distractor, and Stationary Distractor Conditions in Experiment 2

\begin{tabular}{|c|c|c|c|c|c|c|c|}
\hline Dependent Variables & $M$ & $S D$ & $M$ & $S D$ & $M$ & $S D$ & Statistical Values \\
\hline Initiation time (msec) & 397 & 41 & 356 & 42 & 354 & 38 & $7.12, p<.05$ \\
\hline Movement duration (msec) & 812 & 90 & 728 & 70 & 732 & 77 & $F(1,7)=12.11, p<.001$ \\
\hline \multicolumn{8}{|l|}{ Transport component } \\
\hline Time to peak acceleration (msec) & 260 & 27 & 225 & 23 & 227 & 24 & $F(1,7)=10.03, p<.001$ \\
\hline Time to peak acceleration $(\%)$ & 32 & 4 & 31 & 5 & 31 & 2 & n.s. \\
\hline Time to peak deceleration (msec) & 519 & 55 & 458 & 41 & 453 & 44 & $17.12, p<.0001$ \\
\hline Time to peak deceleration $(\%)$ & 64 & 4 & 63 & 6 & 62 & 6 & n.s. \\
\hline Amplitude peak velocity $(\mathrm{mm} / \mathrm{sec})$ & 621 & 89 & 782 & 112 & 757 & 102 & n.s. \\
\hline Time to maximum grip aperture (msec) & 544 & 53 & 466 & 45 & 469 & 46 & $F(1,7)=37.06, p<.0001$ \\
\hline Time to maximum grip aperture $(\%)$ & 67 & 9 & 64 & 3 & 64 & 4 & $F(1,7)=28.09, p<.0001$ \\
\hline Velocity of finger aperture $(\mathrm{mm} / \mathrm{sec})$ & 314 & 30 & 321 & 32 & 456 & 54 & $F(1,7)=45.73, p<.0001$ \\
\hline Amplitude grip aperture $(\mathrm{mm})$ & 86 & 18 & 108 & 11 & 109 & 9 & $F(1,7)=104.54, p<.0001$ \\
\hline
\end{tabular}




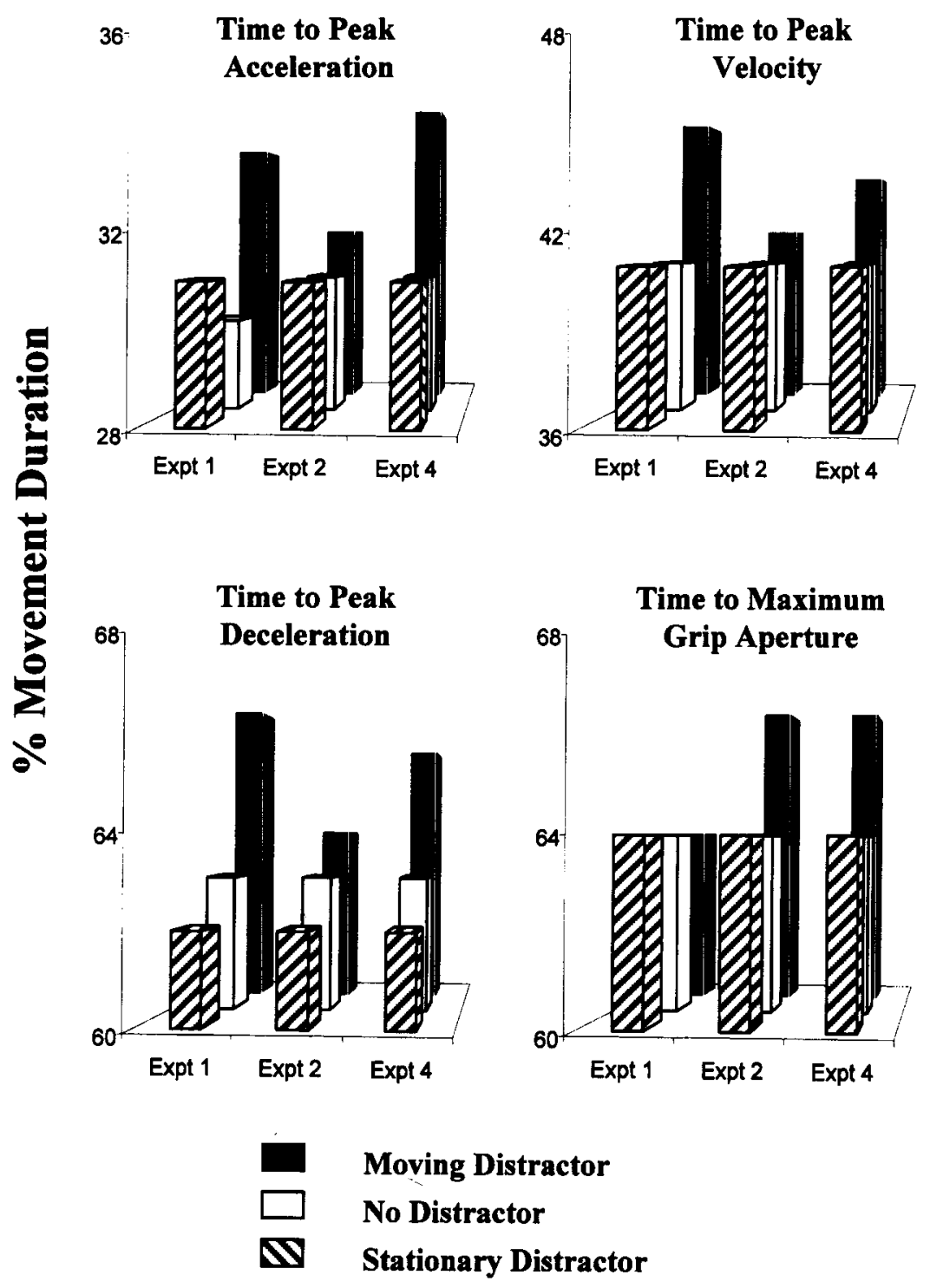

Figure 5. Temporal kinematic parameters expressed as a percentage of movement duration for the three distractor sets for Experiments 1, 2, and 4.

distractor object was positioned in almost the same location as was the fruit target. A similar effect for the manipulation component, but one also associated with changes for the transport component, was found in a previous study (Castiello, 1996). However, in that study, the position of the distractor was at a distance from the position of the target. The eye movement pattern was similar to that found for Experiment 1 (see Figure 3, lower panel). In the moving distractor situation, the eyes moved from the target to the distractor, with deviations in other positions of the visual field.

Further ANOVAs, comparing Experiments 1 and 2, for which experiment ( 1 and 2 ) was the between-subjects factor and type of distractor (stationary and moving) was the within-subjects factor, were carried out. When considered in relative terms, parameters of the transport component - such as the time to peak velocity, acceleration, and deceleration-occurred much later in Experiment 1 than in Experiment 2 for the moving, as compared with the stationary, distractor condition $(p s<.0001$; see Tables 2 and 3 and Figure 5). Conversely, when comparing parameters for the manipulation component-such as the time of maximum grip aperture-it appeared that, in relative terms, it was reached later for Experiment 2 than for Experiment $1[F(1,7)=9.01, p<.001,67 \%$ vs. $64 \%$; see Tables 2 and 3 and Figure 5]. In addition, the differ- 
ence between the maximum finger aperture for Experiment 2, as compared with Experiment 1, for the moving distractor was also significant $[F(1,7)=11.23, p<.001$, 86 vs. $107 \mathrm{~mm}$; see Tables 2 and 3].

Overall, the results from Experiments 1 and 2 suggest that interference occurs on the basis of intrinsic and extrinsic (localization) object characteristics. This distinction between modes of processing the distractor confirms the model proposed by Jeannerod $(1981,1984)$. According to this author, information is processed in two main pathways: the transport channel and a parallel manipulation channel. Hypothetically, in the first experiment, interference effects were present because the channel responsible for processing object localization was overstimulated by the target and the distractor objects. Conversely, as predicted, for Experiment 2, interference effects emerged for object manipulation. This is because it is the channel responsible for preparing the hand to properly grasp the object that was overloaded, given that distractor and target objects competed. This competition was based on the intrinsic object characteristics that dictated different grasp parameterization.

\section{EXPERIMENT 3}

A result of Experiment 2 was the smaller amplitude of the hand aperture for the apple when the raspberry was presented in a moving distractor situation. This result may be ascribed to the possibility of the (smaller) distractor becoming the object. In other words, the type of grasp required by the distractor differs from that required by the target. In particular, the amplitude of grip aperture required by the distractor is much smaller. As a consequence, when there is a possible need to grasp the distractor, subjects adopt a grasp for the target (apple) that is influenced by the probability of grasping the raspberry. The result is smaller finger amplitudes for the target apple in the moving distractor situation. To warrant such a conclusion, an experiment was performed in which a distractor of equal size to the target was presented.

\section{Method}

\section{Subjects}

Eight students ( 4 women and 4 men, 20-24 years of age) volunteered to participate in this experiment; none had participated in the previous experiments. They showed the same general characteristics as did the subjects of the previous experiments. Each subject attended two experimental sessions of 1 -h duration.

\section{Apparatus and Materials \\ The apparatus and materials were the same as those described for Experiment 2. \\ Design \\ The design of the present experiment was similar in all respects to that for Experiment 2, except that the distractor was an apple sim- ilar to the target instead of a raspberry.}

\section{Procedure}

The experiment consisted of two sessions, and two different experimental conditions were tested. The order of sessions was counterbalanced across subjects. Experimentation took place over a 2-day period - one session on Day 1, a second session on Day 2.

Condition A. The procedure for Condition A of the present experiment was in all respects similar to that utilized for Experiment 2, except that both the distractor and the target were apples. Eye movements were again monitored.

Condition B. The procedure and the task for Condition B were exactly the same as those for Condition $A$, but eye movements were not constrained. In other words, the subjects were required to perform exactly the same task as for Condition A, except that they could move their eyes without any limitation.

\section{Results and Discussion}

Initiation time, movement duration, and kinematic variables were analyzed with an ANOVA, with type of distractor (no distractor, moving, stationary) as a withinsubjects factor. For this experiment, when the eye movements were not constrained, no significant effects for any of the dependent measures were found. Only results related to Condition A will be reported. Given the control nature of this experiment, a full account of the results will not be reported. Four cases in which the subjects had to reach for the distractor were registered and later analyzed (see the Appendix). As was found for Experiment 2, initiation time and movement duration were longer for the moving condition than for the stationary condition [initiation time: $F(1,7)=10.12, p<.001,367$ vs. $351 \mathrm{msec}$; movement duration: $F(1,7)=8.21, p<.05,798$ vs. $752 \mathrm{msec}$ ].

In contrast to what was found for Experiment 2, when the eyes were fixated, peak of grip aperture and velocity of finger aperture, measured for the moving distractor, were similar to those measured for the stationary distractor situation (peak grip aperture: 107 and $105 \mathrm{~mm}$, $F(1,7)=0.99, p=.33$; velocity finger aperture: $438 \mathrm{vs}$. $442 \mathrm{~mm} / \mathrm{sec}, F(1,7)=1.11, p=.34$ ). Thus, the results for Experiment 2 suggest that the different size of grip aperture required by the distractor influenced the grasp for the target object.

\section{EXPERIMENT 4}

In Experiments 1, 2, and 3, intrinsic and extrinsic distractor features affected either the transport or the manipulation component. In addition, it emerged clearly that the distractor object, which potentially could become the target, produced interference effects.

These results highlight not only that manipulating the distractor can affect selectively the transport or the manipulation component but also that the time course of interference differs in relation to the different components.

To investigate whether the selective effects found for the two components are additive or nonadditive, a situation in which the distractor involves both the transport 
and the manipulation components was created. We used a paradigm in which the electric carriage displaced a distractor object that differed from the target object in terms of size.

If the systems are completely independent, it is unlikely that perturbing both components together would lead to a greater disruption than adding up the responses to the perturbation of each system individually. In independent systems, the behavior of one system should not affect the behavior of another. In contrast, if an augmented interference is found, the behavior of one system in response to perturbation might depend on whether the other system is simultaneously perturbed or not. In this case, the two systems cannot be independent, as was postulated by Jeannerod $(1981,1984)$.

\section{Method}

\section{Subjects}

Ten students ( 5 women and 5 men, 21-25 years of age) volunteered to participate in this experiment; none had participated in the previous experiments. They showed the same general characteristics as did the subjects of the previous experiments. Each subject attended two experimental sessions of 1 -h duration.

\section{Apparatus and Materials \\ This experiment used the same apparatus as that described in} Experiment 1.

\section{Design}

The design for this experiment was similar to that for Experiment 1, except that the carriage displaced a distractor object (a raspberry) that differed from the target object (an apple).

\section{Procedure}

The procedure and the number of trials were the same as those in Experiment 1 .

Condition A. This condition was a replication of Condition A for Experiment 1, except that the carriage displaced a distractor object (a raspberry) that differed from the target object (an apple). Thus, in the case of perturbed trials, the subject had to implement a different program for the transport and the manipulation components. For this condition, eye movements were monitored.

Condition B. Condition B replicated Condition A, except that eye movements were not constrained.

\section{Results and Discussion}

When the eye movements were not constrained, no significant effects for any of the dependent measures were found. For this reason, in the following section, only the results related to the condition in which the eyes were monitored will be reported. An ANOVA with distractor (no distractor, moving, stationary) as a withinsubjects variable was performed on the dependent measures of interest. No differences between the stationary and the no-distractor conditions were found. Thus, for the sake of brevity, only the values for the moving versus the stationary distractor conditions will be reported in the text. For the other values and statistics, refer to Table 4 . There were no cases in which the subject reached for the distractor.

Both the transport and the manipulation components were affected by the moving distractor. For example, ini- tiation time was longer when the distractor was moving, as compared with stationary (412 vs. $364 \mathrm{msec})$. Also, movement time was influenced by the moving distractor. The time taken to reach and grasp the stationary target object in the presence of a moving distractor object was longer than the time taken to reach and grasp the stationary target object in the presence of a stationary distractor object ( 838 vs. $753 \mathrm{msec}$ ). The time to peak velocity, acceleration, and deceleration, occurred later for the moving than for the stationary distractor condition, both in absolute and relative terms (time to peak velocity: absolute, 368 vs. $308 \mathrm{msec}$; relative, $44 \%$ vs. $41 \%$; time to peak acceleration: absolute, 293 vs. $233 \mathrm{msec}$; relative, $35 \%$ vs. $31 \%$; time to peak deceleration: absolute, 553 vs. $467 \mathrm{msec}$; relative, $66 \%$ vs. $62 \%$ ). Also, the amplitudes of the velocity, acceleration, and deceleration peaks were all lower for the movements performed in the presence of the moving distractor than for those performed in the presence of the stationary distractor (see Table 4). As an example, peak velocity was 554 and $876 \mathrm{~mm} / \mathrm{sec}$, respectively. For the manipulation component, as found in Experiment 2, the maximum amplitude and velocity of grip aperture for the moving distractor situation were smaller than those for the stationary distractor (amplitude grip aperture, 76 vs. $110 \mathrm{~mm}$; velocity of finger aperture, 374 vs. $434 \mathrm{~mm} / \mathrm{sec}$ ).

Overall, these results confirm that information accessed in the implementation of the reach-to-grasp program comes from different characteristics of the distractor. In addition, comparison analyses with experiment (1, $2,4)$ as a between-subjects factor and type of distractor (moving, stationary, no distractor) demonstrate that, in the case of the simultaneous stimulation of both transport and manipulation channels, the level of interference is augmented with respect to perturbation of only one channel. However, this increase was confined to movement duration $[F(1,7)=35.76, p<.0001$; see Figure 6]. In other words, the increase in movement duration observed in Experiment 4 suggests that the distractor effects shown in Experiments 1 and 2 are nonadditive.

\section{GENERAL DISCUSSION}

In the present study, two issues were at stake. One was variation in the reach-to-grasp organization when overt and covert mechanisms of attention were dissociated. The second issue was to investigate whether such a dissociation produced a selective and separate interference effect on the two hypothesized channels (transport and manipulation) that subserve the reach-to-grasp movement (Jeannerod, 1981, 1984).

The results of the present experiments can be summarized as follows. When eye movements and covert attention were dissociated - that is, eye movements remained stationary on the target and covert attention was diverted toward a distractor object-performance was affected. The reach-to-grasp action was modified with respect to cases in which eye movements and covert attention were not subjected to any constraint. However, interference 
Table 4

Initiation Time, Movement Duration, and Kinematic Parameters in the Moving Distractor, No-Distractor, and Stationary Distractor Conditions in Experiment 4

\begin{tabular}{|c|c|c|c|c|c|c|c|}
\hline \multirow[b]{2}{*}{ Dependent Variables } & \multicolumn{2}{|c|}{$\begin{array}{c}\text { Moving } \\
\text { Distractor }\end{array}$} & \multicolumn{2}{|c|}{$\begin{array}{c}\text { No } \\
\text { Distractor }\end{array}$} & \multicolumn{2}{|c|}{$\begin{array}{l}\text { Stationary } \\
\text { Distractor }\end{array}$} & \multirow[b]{2}{*}{ Statistical Values } \\
\hline & $M$ & $S D$ & $M$ & $S D$ & $M$ & $S D$ & \\
\hline Initiation time (msec) & 412 & 45 & 366 & 32 & 364 & 41 & $F(1,9)=36.12, p<.0001$ \\
\hline Movement duration (msec) & 838 & 85 & 749 & 75 & 753 & 76 & $F(1,9)=19.45, p<.0001$ \\
\hline \multicolumn{8}{|l|}{ Transport component } \\
\hline Time to peak velocity (msec) & 368 & 46 & 307 & 34 & 308 & 33 & $F(1,9)=41.04, p<.0001$ \\
\hline Time to peak velocity $(\%)$ & 44 & 5 & 41 & 5 & 41 & 4 & $F(1,9)=36.11, p<.0001$ \\
\hline Time to peak acceleration (msec) & 293 & 29 & 232 & 21 & 233 & 22 & $F(1,9)=11.79, p<.001$ \\
\hline Time to peak acceleration $(\%)$ & 35 & 4 & 31 & 3 & 31 & 4 & $F(1,9)=10.04, p<.001$ \\
\hline Time to peak deceleration (msec) & 553 & 54 & 471 & 44 & 467 & 45 & $F(1,9)=18.12, p<.0001$ \\
\hline Time to peak deceleration (\%) & 66 & 4 & 63 & 6 & 62 & 6 & $F(1,9)=11.23, p<.001$ \\
\hline Amplitude peak velocity $(\mathrm{mm} / \mathrm{sec})$ & 554 & 67 & 845 & 86 & 876 & 89 & $F(1,9)=43.74, p<.0001$ \\
\hline Amplitude peak acceleration $\left(\mathrm{mm} / \mathrm{sec}^{2}\right)$ & 3,825 & 437 & 6,288 & 656 & 6,380 & 589 & $F(1,9)=42.14, p<.0001$ \\
\hline Amplitude peak deceleration $\left(\mathrm{mm} / \mathrm{sec}^{2}\right)$ & 3,456 & 412 & 5,628 & 576 & 5,578 & 587 & $F(1,9)=29.06, p<.0001$ \\
\hline \multicolumn{8}{|l|}{ Manipulation component } \\
\hline Time to maximum grip aperture (msec) & 561 & 58 & 479 & 48 & 482 & 46 & n.s. \\
\hline Time to maximum grip aperture (\%) & 67 & 10 & 64 & 6 & 64 & 5 & n.s. \\
\hline Velocity of finger aperture $(\mathrm{mm} / \mathrm{sec})$ & 374 & 43 & 367 & 33 & 434 & 59 & $F(1,9)=8.03, p<.05$ \\
\hline Amplitude grip aperture (mm) & 76 & 9 & 110 & 9 & 110 & 9 & $F(1,9)=74.38, p<.0001$ \\
\hline
\end{tabular}

emerged only in the moving distractor condition and not in the stationary distractor condition. In the latter case, the distractor could be ignored, given that the execution of the task did not require its appraisal. Following the dissociation between eye movements and attention, the transport and the manipulation components were selectively affected by specific features of the distractor. The transport component was mainly affected when the distractor had to be monitored covertly and assessed for possible changes in its position. The manipulation component was mainly affected when the distractor had to be monitored covertly and assessed for possible changes in size. When the distractor required changes in both the transport and the manipulation components, interference effects to both the transport and the manipulation components were larger than when the distractor induced changes in only one component. However, the locus of interference varied in relation to the type of distractor manipulation, and not only because of the covert/overt dissociation.

The finding that interference effects were found when covert attention was presumably tracking the distractor suggests that an orienting reaction, such as an attentional shift, occurs as a precursor to selection for action mechanisms. This involves heightening of sensitivity in response to the newness, importance, and intensity of the stimulus attributes. This argues for the early identification of salient attributes that are pertinent to potential motor outputs, before they are actually selected as goals for related actions. Thus, registration of significance is accomplished without the need to process all the information available (Bernstein, 1967). This interpretation, however, is based on the assumption that subjects fix their attention on the distractor while they fix their eyes on the target. Nevertheless, it could also be possible that attention is coupled to the eyes and that, therefore, inter- ference arises only when the distractor is processed outside the focus of attention.

In this connection, Castiello (1996) found that, when the focus of attention was directed to a nontarget object, characteristics of this object became relevant and influenced the motor output directed to the target object. In other words, the presence and the nature of the distractor affected both reach and manipulation, indicating that distractor interference during selective reaching is in-

\section{Movement Duration}
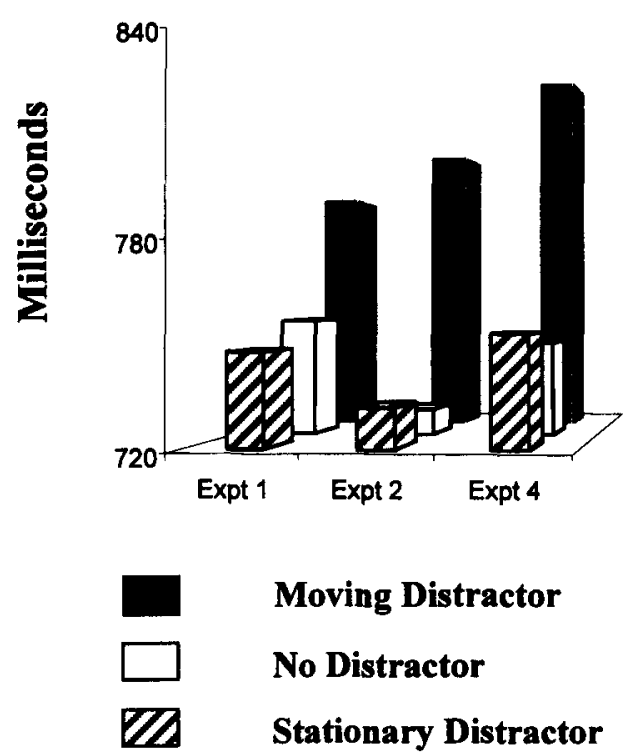

Figure 6. Movement duration for the moving, no-distractor, and stationary sets for Experiments 1, 2, and 4. 
creased when the distractor receives more attention. These results are confirmed in the present study. However, these results differ from the Castiello study in that the manipulation used in the present experiments was more effective in some cases for certain parameters, in some cases for other parameters. Thus, attentional shift toward the distractor not only selects the relevant object characteristics but also directs such information to the visuomotor channels that may require modification. This effect can be interpreted in such a way that there is a need for the allocation of attention to the target position before arm movement is made. Schneider (1995) has proposed a neurocognitive model for functions and mechanisms of visual attention in the primate brain, called the visual attention model (VAM). According to this model, the visual attention mechanism has two functions-namely, the selection of information from an object for spacebased motor actions and the selection of information from the same object for visually based object recognition. In other words, whenever visual attention is allocated to an object, this object can be recognized, and its spatial parameters are computed for eventual motor actions, such as saccades or grasping. The relevance of VAM for the present study is that, when visuospatial mechanisms are directed toward a distractor object, characteristics of that object may be computed and interfere with those established for the target object.

Recently study, Deubel et al. $(1996,1998)$ investigated the role of visual selective attention in the preparation of aiming manual movements. From their results, they concluded that it is not possible to maintain attention on a stimulus while directing a manual movement to a spatially separate object. Rather, they argue for an obligatory and selective coupling of visual attention and movement programming. In our experiments, eye movements were separated from attention; however, attention was not confined singularly to the target but was divided between the target and the distractor, given that, for a successful performance, the subjects had to monitor both objects. This difference could account for the different results obtained in Deubel et al. and those of our studies - that is, performance deterioration in the former case and selective interference effects that do not lead to movement breakdown in the latter. Alternatively, since no speeded response to the target was required, a possible strategy for the subject could have been to monitor only the distractor but not the target by covert or, in Condition B, overt attention. Thus, attention was shifted to the target only when required. In this case, the subject would first prepare a grasp to the distractor (because attention was directed to this object), and the interference effects could be interpreted as being reminiscent of the old motor program.

Of relevance for the present study is that, as each object provides both extrinsic information related to the kinematic parameter of the reach and intrinsic information related to the kinematic parameter of the grasp, any mechanism underlying a choice might simplify this volume of complex visual information. Recollect that, in the present experiments, at any time the distractor object could become the target. From the results, it appears that, once attention is oriented toward the distractor, it extracts only that information necessary to accomplish a possible selective change in motor programming.

The present study provides a confirmation of the chan$n e l$ hypothesis proposed by Jeannerod $(1981,1984)$. The fact that the distractor acts selectively on each component individually suggests that attention selects object attributes relevant for a possible change in the visuomotor channel required for the eventual modification. That is, spatial attributes are taken into account when it is mainly the transport component that needs to be varied. Intrinsic attributes, such as shape and size, are taken into account when it is mainly the manipulation component that needs to be varied.

Thus, it appears that the idea of the two independent channels is supported by empirical evidence. For example, in Experiment 1, the distractor was an apple that moved laterally behind the target (another apple) and that sometimes could change direction toward the target, thus becoming the to-be-grasped object. In this case, only the reach component was affected by the experimental manipulation, because target and distractor had, eventually, to be reached in different positions, whereas the manipulation component for the two objects remained the same. Conversely, in Experiment 2, in which the distractor was a stationary piece of fruit (a raspberry) that sometimes became the target object because of a sudden change in illumination, interference effects were observed with the grasp component but not with the reach component, given that the two objects were in close proximity. Thus, it can be suggested that, whereas the reaching component did not require changes, the manipulation component required an alteration, considering the different type of grasp parameterization. However, for Experiment 4, in which the distractor required modification at both the transport and the manipulation levels, the nonadditive results point toward interdependence, and interference was evident for both components. In general, interference emerged from the competition between channels that were simultaneously stimulated by the target and the distractor. In other words, adding a distractor that is task relevant along two dimensions may result in sufficient interference to affect both channels.

Looking at the results more closely, it appears that the presence of the moving distractor affects kinematics at different stages for Experiments 1 and 2. For Experiment 1 , the kinematic parameters for the transport component are delayed. For Experiment 2, the kinematic parameter for the manipulation component are delayed. These latter changes occur later than the former. This resembles previous results obtained from studies that have investigated the consequences of perceptual perturbations on the reach-to-grasp movement (Castiello et al., 1991; Paulignan, Jeannerod, MacKenzie, \& Marteniuk, 1991; Paulignan, MacKenzie, et al., 1991; for a review, see Haggard, 1994). In these studies, the time that elapses 
before the first correction begins is significantly different when perturbation signals a change in the manipulation or in the transport component. When the perturbation implies changes in the grasp component, the adjustments occur after about $300 \mathrm{msec}$, considerably later than the adjustments following perturbation of object location, which occur after about $100 \mathrm{msec}$. A likely explanation for these results could be that the perceptual processing required to adapt the motor output to the sudden change of object semantic attributes is more demanding than the one needed to compute its changed spatial position. The visual stimulus provided by a change in object position is most salient and may be strongly represented in fast subcortical visual pathways, such as those involving the superior colliculus, which are known to be involved in producing rapid orienting responses toward changing targets. Changes in object size or shape might involve slower visual cortical pathways, which represent the intrinsic feature of the object (Haggard, 1994). As stated by Jeannerod (1994), semantic processing is more complex, because it requires binding into a single, identifiable, and meaningful entity the many elementary attributes of the object that have been processed in different neural pathways. Processing the spatial localization of a stimulus, instead, does not imply binding of object attributes into a single entity, so it is considered less complex. This fact could account for both the results of the perturbation studies and the results of our experiments.

The explanation suggested to justify the different interference effects found in Experiment 1 and Experiment 2 is also supported by the results of Experiment 3 . In that case, the distractor was the same object as the target (an apple), and no interference on the kinematic parameterization of the movement was found. This result confirms that the findings for Experiment 2 have to be ascribed to the possibility of the (smaller) distractor becoming the object, thus implying a different semantic processing. In Experiment 3, instead, the distractor was an equally sized object that needed no further semantic processing. Thus, it appears that the results described for Experiment 2 are related to the possibility that the cognitive computations underlying the different size of prehension required by the distractor influenced the grasp for the target object apple.

When looking at the results of Experiment 4, intrinsic kinematic parameterization is affected for both the transport and the manipulation component. Thus, the double distractor object stimulation in both spatial and semantic terms seems to affect both channels. Possibly, this occurs because both transport and manipulation systems are overstimulated. The result is a retardation at the final motor output stage.

In conclusion, the present results could provide an account of how covert attention and eye movements are organized to produce an efficient performance. It is suggested that, independently from the amount of attention to the target before arm movement is initiated, motor output optimization is reached when eye movements are also directed to the target. However, this does not mean that covert attention by itself is not enough to guide a correct movement. In fact, the interference effects found in the present series of experiments do not result in a complete breakdown in movement, such as missing the target. They produce a slowness and a reorganization of the kinematic parameterization of the movement, which give us clues as to how movements are programmed.

\section{REFERENCES}

BERNSTEIN, A. S. (1967). The coordination and regulation of movement. Oxford: Pergamon.

Bootsma, R. J., \& VAN Wieringen, P. C. W. (1992). Spatio-temporal organization of natural prehension. Human Movement Science, 11, 205-215.

CASTIELlo, U. (1996). Grasping a fruit: Selection for action. Journal of Experimental Psychology: Human Perception \& Performance, 22, 582-603.

Castielloo, U., Bennett, K. M. B., \& Stelmach, G. E. (1993). Reach to grasp: The natural response to a perturbation of object size. Experimental Brain Research, 94, 165-178.

Castiello, U., Paulignan, Y., \& Jeannerod, M. (1991). Temporal dissociation of motor responses and subjective awareness. Brain, 114, 2639-2655.

D'Amico, M., \& Ferrigno, G. (1990). Technique for the evaluation of derivatives from noisy biomechanical displacement data using a model-based bandwidth-selection procedure. IEEE Transactions on Biomedical Engineering, 28, 407-415.

D'Amico, M., \& Ferrigno, G. (1992). Comparison between the more recent techniques for smoothing and derivative assessment in biomechanics. IEEE Transactions on Biomedical Engineering, 30, 193-204.

Deubel, H., Schneider, W. X., \& Paprotta, I. (1996). Visual attention and manual aiming: Evidence for obligatory and selective spatial coupling. Perception, 25, 13-14.

Deubel, H., Schneider, W. X., \& Paprotta, I. (1998). Selective dorsal and ventral processing: Evidence for a common attentional mechanism in reaching and perception. Visual Cognition, 5, 81-90.

DunCan, J., \& DESIMONE, R. (1995). Neural mechanisms of visual spatial attention. Annual Review of Neuroscience, 18, 193-222.

Ferrigno, G., \& Pedotti, A. (1985). ElitE: A digital dedicated hardware system for movement analysis via real-time TV signal processing. IEEE Transactions on Biomedical Engineering, 32, 943-950.

Gentilucci, M., Castiello, U., Corradini, M. L., Scarpa, M., Umiltà, C., \& Rizzolatti, G. (1991). Influence of different types of grasping on the transport component of prehension movements. Neuropsychologia, 29, 361-378.

Gentilucci, M., Chieffi, S., Scarpa, M., \& Castiello, U. (1992). Temporal coupling between transport and grasp components during prehension movements: Effects of visual perturbation. Behavioural Brain Research, 47, 71-82.

HAGGARD, P. (1994). Perturbation studies of coordinated prehension. In K. M. B. Bennett \& U. Castiello (Eds.), Insights into the reach to grasp movement (pp. 151-170). Amsterdam: North-Holland.

HAGgaRd, P., \& WiNG, A. (1990). Assessing and reporting the accuracy of position measurements made with optical tracking systems. Journal of Motor Behavior, 22, 315-321.

JAKOBSON, L. S., \& GoODALE, M. A. (1991). Variables affecting higherorder movement planning: A kinematic analysis of human prehension. Experimental Brain Research, 86, 199-208.

JEANNEROD, M. (1981). Intersegmental coordination during reaching at natural visual objects. In J. Long \& A. Baddeley (Eds.), Attention and Performance IX (pp. 153-169). Hillsdale, NJ: Erlbaum.

JEANNEROD, M. (1984). The timing of natural prehension movements. Journal of Motor Behavior, 16, 235-254.

JEANNEROD, M. (1994). Object oriented action. In K. M. B. Bennett \& U. Castiello (Eds.), Insights into the reach to grasp movement (pp. 315). Amsterdam: North-Holland.

Marteniuk, R. G., Leavitt, J. L., Mackenzie, C. L., \& Athenes, S. (1990). Functional relationships between the grasp and transport components in a prehension task. Human Movement Science, 9, 149-176. 
OldFieLD, R. C. (1971). The assessment and analysis of handedness: The Edinburgh Inventory. Neuropsychologia, 9, 97-113.

Paulignan, Y., Jeannerod, M., MacKenzie, C. L., \& MarteNIUK, R. G. (1991). Selective perturbation of visual input during prehension movements. 2: The effects of changing object size. Experimental Brain Research, 87, 407-420.

Paulignan, Y., Mackenzie, C. L., Marteniuk, R. G., \& JeanNEROD, M. (1991). Selective perturbation of visual input during prehension movements. 1: The effects of changing object position. $E x$ perimental Brain Research, 83, 502-512.

PoSNER, M. I. (1980). Orienting of attention. Quarterly Journal of Experimental Psychology, 32, 3-25.

SCHNEIDER, W. X. (1995). VAM: A neuro-cognitive model for visual attention control of segmentation, object recognition and space-based motor actions. Visual Cognition, 2, 331-376.

UMILTÀ, C. (1988). Orienting of attention. In F. Boller \& J. Grafman (Eds.), Handbook of neuropsychology (Vol. 1, pp. 175-193). Amsterdam: Elsevier.

WALLACE, S. A., \& WEEKS, D. L. (1988). Temporal constraints in the control of prehensile movements. Journal of Motor Behavior, 20, 81-105.

Wing, A. M., TurTon, A., \& Fraser, C. (1986). Grasp size and accuracy of approach in reaching. Journal of Motor Behavior, 18, 245-260.

\section{APPENDIX}

In the present Appendix, those trials for which the subjects had to reach for the distractor will be described. These cases occurred only for Experiments 1 and 3 . Given the limited number of trials, they will just be analyzed from a qualitative point of view.

\section{Experiment 1}

For some cases during the moving distractor situation, the subjects grasped the distractor object after it crossed the red mark. These trials were 12 out of 800 and were not related to the peculiar performance of only 1 subject. In particular, Subject 2 had 3, Subject 4 had 1, Subject 5 had 2, Subject 6 had 2, Subject 7 had 3, and Subject 8 had 1.

It is difficult to identify a common pattern for these trials, even for those performed by the same subject. Two main types of such patterns will be described.

Type 1 (five trials). After departure, the reaching movement was reaccelerated, and this results in a double step pattern. This was evident on the double peak velocity profile. Such a reacceleration did not produce any effect, either on the transport component or on the manipulation component.

Type 2 (four trials). Occasionally, for some of the trials in which the carriage was programmed to turn, the subjects waited too long before leaving the starting switch, thus allowing the distractor to cross over the red mark. When this was the case, the subjects went straight for the apple distractor, and their movements did not show any difference.

\section{Experiment 3}

In the moving distractor condition, Subjects $3,4,7$, and 8 reached once for the distractor apple, instead of reaching for the target apple. For these trials, no particular pattern in the kinematics, with respect to the trials directed to the target, was found.

(Manuscript received May 5, 1997;

revision accepted for publication November 16, 1997.) 